\title{
Imaging flow cytometry enhances particle detection sensitivity for extracellular vesicle analysis
}

\author{
The study of extracellular vesicles (EVs) has evolved into a compelling field of intercellular communication \\ and pathophysiology research. The submicron size of EVs and the complexity of the biofluids in which they \\ are suspended confound their analysis by traditional light microscopy or flow cytometry. Observation and \\ quantitation of EVs have been achieved with Amnis ${ }^{\circledR}$ imaging flow cytometers (IFCs) with reduced volumes \\ and sample preparation times, suggesting an auspicious role for IFCs in the advancement of EV biology.
}

\section{EVs: the rich frontier between cells and debris}

Few areas of biomedical research have generated more interest in the past decade than EV biology. Secreted by most, if not all, human cell types in response to activation, injury or apoptosis, EVs include microvesicles, exosomes and apoptotic bodies. Formed intracellularly or shed by budding of the plasma membrane, these tiny membranebound packages of enigmatic macromolecules once overlooked as cellular debris are now recognized as key emissaries mitigating cell-cell interactions. In fact, EVs may be the principal means for the transfer of genetic material and signaling molecules among cells in interstitial spaces and via biological fluids such as plasma, cerebrospinal fluid, sputum and milk. This capacity to ferry bioactive molecules among cells and tissues has underscored the potential significance of EVs in fields as diverse as hematology, regenerative medicine, vascular disease, inflammation and oncology. Here we describe how Amnis ${ }^{\circledR}$ IFCs provide a uniquely sensitive platform for the direct detection of EVs while reducing sample volume and preparation time.

\section{Small-scale biology = large-scale technical challenges}

Because of the small size of EVs and the variable nature of the biofluids in which they are typically suspended, disparate approaches to their collection, isolation and analysis have led to efforts to enhance the accuracy and correlation of data. Although EVs' clinical presentation in biofluids and in cell-conditioned culture media suggests that flow cytometry could be used as a platform for analysis, their size (generally 50-1,000 nm in diameter) presents technical challenges for both traditional, photomultiplier tube (PMT)-based flow cytometers and light microscopy. These limitations hinder efforts to come to a consensus regarding applications for the assessment of $\mathrm{EVs}$, and concerns about the comparability of data have been raised among EV researchers.

\section{Robin T Clark}

EMD Millipore Corporation, Billerica, Massachusetts, USA. Correspondence should be addressed to R.T.C. (robin.clark@emdmillipore.com).

\section{Automating reliable quantitation of submicron particles}

Definitive detection of EVs and determination of their absolute numbers per volume of suspension are critical but have proven to be technically challenging with traditional flow cytometers. The Amnis ${ }^{\circledR}$ FlowSight ${ }^{\circledR}$ and ImageStream ${ }^{\circledR X}$ instruments collect both image and fluorescence-intensity data by means of a CCD (charge-coupled device) camera. This technology quickly demonstrated superior fluorophore emission sensitivity in tests of the ability of the CCD system to resolve dim and rare populations in the context of leukocyte heterogeneity in blood samples (Fig. 1).

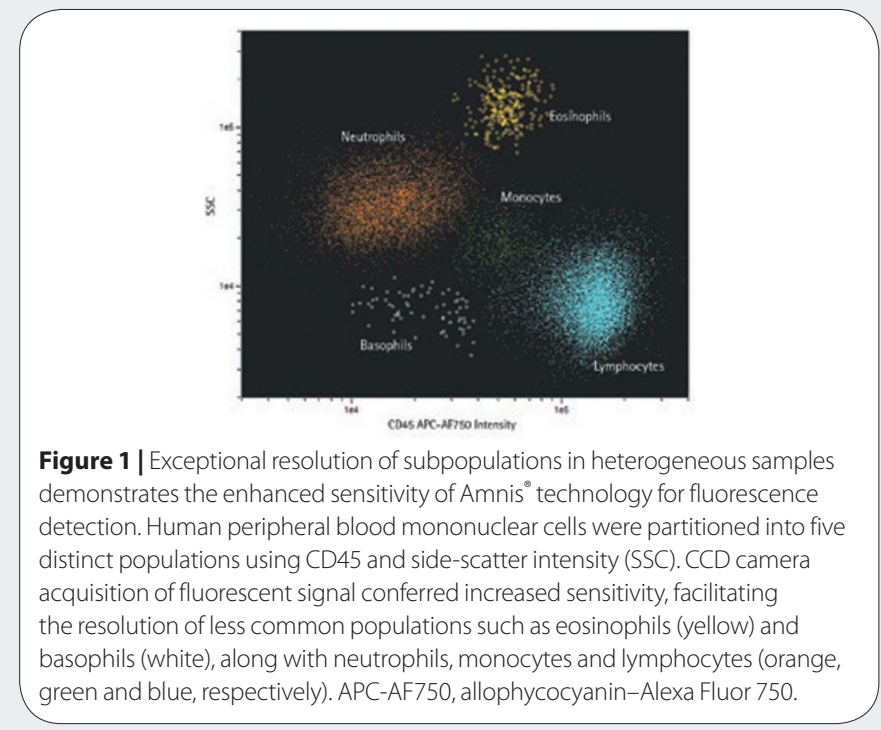

Because the event count and volume acquired per sample are automatically recorded by the Amnis INSPIRE ${ }^{\circledR}$ acquisition software, the number of EVs per microliter may be readily displayed within the IDEAS ${ }^{\circledR}$ analysis statistical workspace. This capability addresses recommendations of the International Society for Extracellular Vesicles regarding the standardization and reduction of workflows in EV preparation and analysis. 
Image-quantitation features enhance vesicle gating strategy Gating with non-imaging flow cytometers relies on the degree of light scatter and fluorescence intensity, which leads to the frequent, inadvertent inclusion of contaminating particles in the presumed EV population. IFCs exploit the ability to connect the quantitative morphological characteristics of an object to its image in the image gallery to confirm and refine gating. This capability has already led to the confirmation of some existing EV gating strategies, while challenging others.

The CCD-based detection used in the Amnis ${ }^{\circledR}$ systems is ideal for the investigation of particles significantly smaller than the 400-500$\mathrm{nm}$ threshold of even the most sensitive PMT-based cytometers (Fig. 2). In addition to offering features that enhance the accuracy and reproducibility of microparticle data, Amnis ${ }^{\circledR}$ systems allow significantly reduced sample-preparation times. Vesicles, cells and beads in heterogeneous suspension are efficiently distinguished by side-scatter and morphology characteristics, eliminating the need to isolate EVs from parent cells or beads prior to flow acquisition.
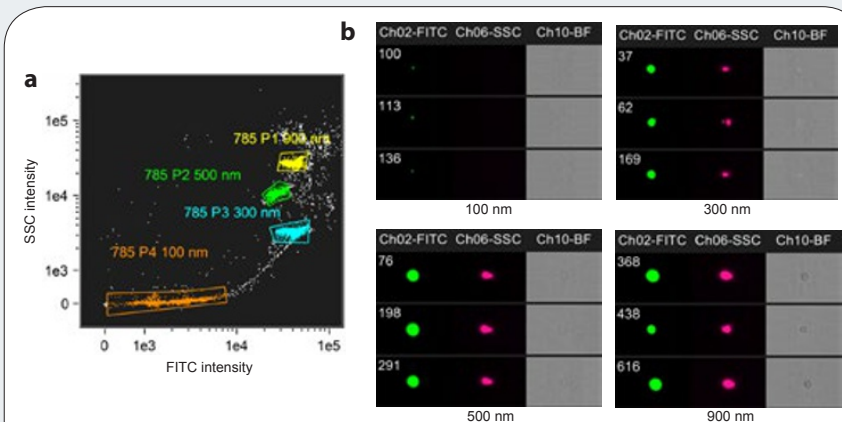

Figure $\mathbf{2}$ | Scatter plots and images of FITC-conjugated beads validate the Amnis ImageStream ${ }^{\circ} \mathrm{x}$ for submicron-particle detection. A mixture of fluorescent beads of known size $(900,500,300$ and $100 \mathrm{~nm})$ was analyzed on an ImageStream ${ }^{\circ}$ instrument using a $60 \times$ objective. (a) A scatter plot of SSC (785$\mathrm{nm}$ detection) versus FITC intensity resolves four discrete populations. (b) FITC, SSC and brightfield (BF) imagery confirm intensity data from bead populations. FITC staining is evident on all beads, from 900 to $100 \mathrm{~nm}$.

\section{Precise fluidics and image confirmation}

Equipped with a $60 \times$ objective, the Amnis ImageStream ${ }^{\circledR X}$ has demonstrated a capacity for the detection and characterization of extracellular microparticles, primarily owing to technological features that distinguish Amnis ${ }^{\circledR}$ instruments (see refs. 1-5 and Fig. 3). First, the barriers to resolving individual microvesicles in biofluids are addressed by exceptionally precise hydrodynamic focusing, which reliably segregates EVs as individual acquisition events. Second, fluorescenceintensity data are paired to an image for every event collected, creating searchable scatter plots and image galleries that facilitate visual confirmation of fluorescent events.

\section{Pioneering biology requires leading-edge technology}

EVs are now believed to be significant mediators in the pathogenesis of neurological, vascular, hematological and autoimmune disease. Moreover, EVs are considered key to tumorigenesis, metastasis, immunomodulation and other cancer processes that may contribute to poor disease outcomes. In order for the assessment of EVs to transition into the clinic to aid in diagnosis and treatment, it is essential to identify technologies that will facilitate the development of standard assays for quantitation and characterization. Although sensitive enough to resolve tiny cellular particles, high-magnification microscopy is limited by the typical dispersal of microvesicles in biofluids, as well as by a lack of quantitative data. Non-imaging flow cytometers are well suited for measuring objects in suspension but are generally unable to detect particles less than $400 \mathrm{~nm}$ in diameter, and they cannot provide visual confirmation of particle integrity for confident characterization.

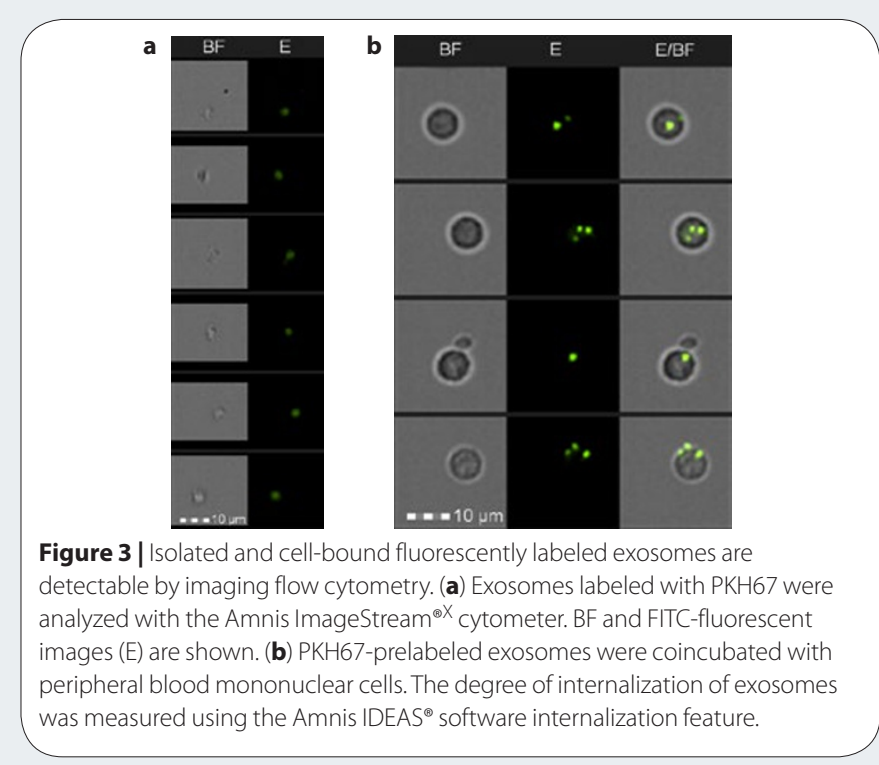

Through precise fluid mechanics, state-of-the-art fluorescencedetection technology, and thoughtful software engineering that facilitates quantitation and statistical analysis, Amnis ${ }^{\circledR}$ IFCs overcome technological barriers to accurate EV assessment. Direct linkage of every event collected to a corresponding image provides additional functionality that allows the user to distinguish true single particles from aggregates and cellular debris. Together, these engineering innovations enable a new gold standard for the consistent detection, quantitation and characterization of EVs on the basis of morphology as well as the expression of cell-surface receptors and other markers.

1. Erdbrügger, U. et al. Imaging flow cytometry elucidates limitations of microparticle analysis by conventional flow cytometry. Cytometry A $\mathbf{8 5}, 756$ 770 (2014).

2. Headland, S.E., Jones, H.R., D'Sa, A.S., Perretti, M. \& Norling, L.V. Cutting-edge analysis of extracellular microparticles using ImageStream ${ }^{X}$ imaging flow cytometry. Sci. Rep. 4, 5237 (2014)

3. van der Meel, R., Krawczyk-Durka, M., van Solinge, W.W. \& Schiffelers, R.M. Toward routine detection of extracellular vesicles in clinical samples. Int. J. Lab. Hematol. 36, 244-253 (2014).

4. Venable, A.S., Williams, R.R., Haviland, D.L. \& McFarlin, B.K. An analysis of endothelial microparticles as a function of cell surface antibodies and centrifugation techniques. J. Immunol. Methods 406, 117-123 (2014).

5. Witwer, K.W. et al. Standardization of sample collection, isolation and analysis methods in extracellular vesicle research. J. Extracell. Vesicles $\mathbf{2}$, http://dx.doi. org/10.3402/jev.v2i0.20360 (2013).

This article was submitted to Nature Methods by a commercial organization and has not been peer reviewed. Nature Methods takes no responsibility for the accuracy or otherwise of the information provided. 